\title{
GEOMETRIC CLASSIFICATION OF COMMUTATIVE ALGEBRAS OF ORDINARY DIFFERENTIAL OPERATORS ${ }^{\dagger}$
}

\author{
Motohico Mulase $\neq$ \\ Department of Mathematics \\ and \\ Institute of Theoretical Dynamics \\ University of California \\ Davis, CA 95616
}

Dedicated to the memory of Professor Michio Kuga

1. The purpose of this paper is to give a geometric classification of all commutative algebras consisting of linear ordinary differential operators whose coefficients are scalar-valued functions.

The problem of determining commuting ordinary differential operators has a long history in mathematics - it started in 1879. Since then it has been studied by many people in various contexts and from different motivations. The so-called rank one case (see Section 2 for definition) was essentially worked out in the 1920's and the most general theorem in the rank one case was obtained by Krichever and Mumford in 1970's. We will present here a complete solution of this problem which is valid for all ranks and generalizes naturally the theorem of Krichever in terms of the geometry of vector bundles on algebraic curves.

Let $B$ be a commutative algebra of linear ordinary differential operators with scalar-valued functions as coefficients. We say two operators $P$ and $Q$ commute with one another if the operator product $P \cdot Q$ coincides with $Q \cdot P$. We impose the following conditions on $B$.

(B-1) $B$ is a $\mathbb{C}$-algebra with the identity operator $1 \in B$.

\footnotetext{
${ }^{\dagger}$ Article appeared in Proceedings of the 18-th International Conference on Differential Geometric Methods in Theoretical Physics: Physics and Geometry, ed. by L. L. Chau and W. Nahm, Plenum Publ., 13-27 (1990).

${ }^{\ddagger}$ Research supported by NSF Grant DMS 8610730, The Institute for Advanced Study, and The Aspen Center for Physics.
} 
(B-2) $B$ has an operator $P$ of order $n>0$ such that its leading coefficient is 1 and the other coefficients have power series expansion at one point. In other words, $B$ has a monic operator which is regular at a certain point.

Since we can always add the identity operator to a given commutative algebra, (B-1) is not a restriction for $B$. The second condition (B-2) looks serious. For example, $\mathbb{C}\left[x \frac{d}{d x}\right]$ is a commutative algebra of ordinary differential operators but it does not satisfy (B-2). However, if we change the coordinate $x$ by $u=\log x$, then the operator $x \frac{d}{d x}$ becomes

$$
\begin{aligned}
x \frac{d}{d x} & =x \cdot \frac{d u}{d x} \cdot \frac{d}{d u} \\
& =\frac{d}{d u},
\end{aligned}
$$

which is monic. It means that the notion of monicness depends on the choice of a coordinate. In general, if we use the coordinate $u$ defined by

$$
u=\int f(x)^{-\frac{1}{n}} d x
$$

then $f(x)\left(\frac{d}{d x}\right)^{n}$ becomes a monic $n$-th order differential operator in $u$. Thus it is not a severe condition to require the existence of a monic operator in $B$. We can simply change the coordinate to make one of the operators in $B$ monic. Similarly, if an operator is given in a practical situation, we can find a point at which all the coefficients have power series expansion. Therefore, (B-2) is not a strong restriction, either.

Let us choose a coordinate $x$ so that the operator $P$ is monic and is regular at $x=0$. Thus every coefficient of $P$ is an element of the ring $\mathbb{C}[[x]]$ of formal power series in $x$. We identify two commutative algebras $B_{1}$ and $B_{2}$ if there is an invertible function $f=f(x) \in \mathbb{C}[[x]]$ such that

$$
B_{1}=f \cdot B_{2} \cdot f^{-1}
$$

In this paper, we present a bijective correspondence, or a dictionary, between the set of commutative algebras of ordinary differential operators satisfying (B-1) and (B-2), and a moduli space of certain geometric data consisting of an algebraic curve, a smooth point on it, a vector bundle on the curve, a local covering of the curve defined on a neighborhood of the point and a local trivialization of the bundle near the given point.

Professor Michio Kuga has worked in the area of mathematics where number theory, algebraic geometry and the theory of differential equations meet together $[\mathrm{Ku}]$. After I finished my work on the Schottky problem [M1-2], I spent one year 
at Stony Brook with him. I really enjoyed discussing mathematics and exchanging mathematical dreams with him. Around that time, I started to think about the higher rank analogue of the Krichever construction and the classification problem of the commuting differential operators.

When the final answer to this problem was obtained, I was truly surprised by the deep interplay between algebraic geometry and the theory of differential equations [M3]. Today, I wish I could tell him this result, and ask him about its possible relation to number theory.

2. In order to give the precise statement of the main theorem, let us define the moduli space $\mathcal{M}_{r}^{+}(0,-1)$ of quintuples $(C, p, \mathcal{F}, \pi, \phi)$ which is formed by the following objects:

- $C$ is an irreducible complete algebraic curve, i.e. a compact Riemann surface with (or without) singularities;

$-p$ is a smooth point of $C$;

- $\mathcal{F}$ is a torsion free sheaf of $\mathcal{O}_{C}$-modules of rank $r$ such that

$$
H^{0}(C, \mathcal{F})=H^{1}(C, \mathcal{F})=0 .
$$

If $C$ is a non-singular curve of genus $g$, then $\mathcal{F}$ is a vector bundle on $C$ of degree $r(g-1)$ with no non-trivial holomorphic sections;

$-\pi: U_{0} \rightarrow U_{p}$ is an $r$-sheeted covering ramified only at $p=\pi(0) \in U_{p}$, where $U_{0}$ is an open disk of $\mathbb{C}$ with center at the origin 0 , and $U_{p} \subset C$ is an open neighborhood of $p \in C$;

$-\phi:\left.\mathcal{F}\right|_{U_{p}} \stackrel{\sim}{\longrightarrow} \pi_{*} \mathcal{O}_{U_{0}}(-1)$ is an $\mathcal{O}_{U_{p}}$-module isomorphism, where $\left.\mathcal{F}\right|_{U_{p}}$ is the restriction of the vector bundle $\mathcal{F}$ on $U_{p}$ and $\pi_{*} \mathcal{O}_{U_{0}}(-1)$ is the direct image by the covering map $\pi$ of the twisted line bundle $\mathcal{O}_{U_{0}}(-1)$ on $U_{0}$ defined by the divisor $\{0\}$ of $U_{0}$.

Since $\pi_{*} \mathcal{O}_{U_{0}}(-1)$ is a trivial vector bundle of rank $r$ on $U_{p}$, we can say that $\phi$ gives a local trivialization of $\mathcal{F}$ on $U_{p}$.

We say that two quintets $\left(C, p, \mathcal{F}, \pi_{1}, \phi_{1}\right)$ and $\left(C, p, \mathcal{F}, \pi_{2}, \phi_{2}\right)$ are isomorphic if there is an automorphism $h: U_{0} \stackrel{\sim}{\longrightarrow} U_{0}$ and a line bundle automorphism $\psi$ : $\mathcal{O}_{U_{0}}(-1) \stackrel{\sim}{\longrightarrow} \mathcal{O}_{U_{0}}(-1)$ such that $\pi_{1}=\pi_{2} \circ h$ and $\phi_{1}=\phi_{2} \circ \pi_{*}(\psi)$. Let us denote the set of all isomorphism classes of quintets by $\mathcal{M}_{r}^{+}(0,-1)$. Now we can state the main theorem.

\section{Main Theorem.}

Let $\mathcal{B}_{r}$ be the set of all commutative algebras $B$ of rank $r$ consisting of ordinary differential operators with scalar-valued function as coefficients satisfying (B-1) and (B-2), where the rank of $B$ is defined by

$$
\operatorname{rank} B=G . C . D .\{\operatorname{ord} Q \mid Q \in B\} .
$$

Then there is a natural bijection

$$
\mu_{r}: \mathcal{B}_{r} \stackrel{\sim}{\longrightarrow} \mathcal{M}_{r}^{+}(0,-1)
$$


for every $r>0$.

In other words, there is a bijective correspondence between the following objects: Analytic Object. A commutative algebra of linear ordinary differential operators of rank $r$ satisfying (B-1) and (B-2). Two of such algebras are identified by (1).

Geometric Object. An isomorphism class of a quintet $(C, p, \mathcal{F}, \pi, \phi)$ consisting of a curve, a point, a vector bundle, a local covering and a local trivialization.

Remark. In the case of rank one, the isomorphism relations of the quintets almost completely wipe out the information of $\pi$ and $\phi$. Therefore, $\mathcal{M}_{1}^{+}(0,-1)$ is exactly the same as the moduli space of the data $(C, p, \mathcal{L}, v)$, where $\mathcal{L}$ is a generic line bundle of degree $g-1$ and $v$ is a non-zero tangent vector of $C$ at $p$. Thus our theorem reduces directly to the theorem of Krichever and Mumford [K], [Mum].

Verdier $[\mathrm{V}]$ obtained a different classification of commutative rings of ordinary differential operators. But since he uses the parabolic structure and the connections of the vector bundles, and since he does not incorporate the identification of (1), his result has a rather different flavor from the point of view of the work of Krichever and Mumford.

3. Since the key ideas of the theorem emerged from the earlier works of many mathematicians, let us sketch the history of this problem. We will concentrate on the old part of the history here, because an extensive review of recent works on this subject can be found in [PW].

Since differential operators do not usually commute, it is natural to ask what happens if they do. As far as I know, the first person who asked this question and tried to give a systematic answer was G. Wallenberg. In his paper [W] of 1903, Wallenberg studied the classification problem of pairs of commuting ordinary differential operators. He mentioned that this problem did not seem to be studied before, even in the fundamental work of G. Floquet $[\mathrm{F}]$. But he credited Floquet that the case of two operators of order one has been worked out in $[F]$.

Floquet's 1879 paper [F] is 130-page long and seems to be considered as one of the standard references of ordinary differential equations in his time. Among other things, he established the Euclid division algorithm for ordinary differential operators.

Let $P$ and $Q$ be ordinary differential operators of order $m$ and $n$, respectively. If $m \geq n$ and the leading coefficient of $Q$ is invertible, then there are operators $P^{\prime}$ of order $m-n$ and $R$ of order less than $n$ such that

$$
P=P^{\prime} Q+R
$$

Thus the system

$$
\left\{\begin{array}{l}
P \psi=0 \\
Q \psi=0
\end{array}\right.
$$


is equivalent with

$$
\left\{\begin{array}{l}
Q \psi=0 \\
R \psi=0
\end{array}\right.
$$

which has lower order than the first one. From the modern point of view, we can say that Floquet studied $\mathcal{D}$-modules. In fact, he proved the following: let $D_{K}$ be the set of all ordinary differential operators with coefficients in the field $K=\mathbb{C}((x))$ of formal Laurent resies in $x$. Then every $D_{K}$-module is generated by a single element. Since he was interested in the non-commutative nature of the ring $D_{K}$, he did not ask the question of Wallenberg.

It is easy to determine all commuting operators of order one. Wallenberg started from this point. In $[\mathrm{W}]$, he gave a complete determination of commuting operators $P$ and $Q$ when

1. ord $P=$ ord $Q=2$, and

2. ord $P=1$ and ord $Q=n>0$.

So far still everything is easy. Then he studied the case of ord $P=2$ and ord $Q=$ 3 , and noticed that the Weierstrass elliptic function appears in the coefficients of these operators (cf. Eq. (2)). He dealt with a few more examples such as order 2 and 5, but did not obtain any general theorem. Maybe Wallenberg's attempt was too much ahead of time. Indeed, the first general result toward the classification problem was only established 20 years later.

In 1923, Burchnall and Chaundy [BC] started the systematic investigation on this problem, without knowing the previous work of Wallenberg. One of their remarkable theorems asserts

First Theorem of Burchnall-Chaundy. Let $P$ and $Q$ be linear ordinary differential operators of positive order. If $P$ and $Q$ commute, then they satisfy a non-trivial polynomial relation $f(P, Q) \equiv 0$, where $f(\lambda, \mu) \in \mathbb{C}[\lambda, \mu]$ is a non-zero polynomial in two variables.

Let us illustrate why this is true. So let $P$ be a second order operator and $Q$ be of order three. As we observed in Section 1, we can assume that $P$ is monic. Note that every operator which commutes with a monic operator of positive order must have a constant leading coefficient. Thus the leading coefficient of $Q$ is a constant. Therefore, we can choose a constant $c_{1} \in \mathbb{C}$ such that $Q^{2}-c_{1} P^{3}$ has order less than 6. Since $Q^{2}-c_{1} P^{3}$ commutes with $P$, its leading coefficient is again a constant. So we can find another constant $c_{2}$ so that $Q^{2}-c_{1} P^{3}-c_{2} P Q$ becomes an operator of order less than 5 . We can continue this procedure until we obtain an operator

$$
R=Q^{2}-c_{1} P^{3}-c_{2} P Q-c_{3} P^{2}-c_{4} Q-c_{5} P
$$

of order less than 2. If ord $R<1$, then $R$ must be a constant because it commutes with $P$. If ord $R=1$, then we can choose constants $c_{6}$ and $c_{7}$ such that $P-c_{6} R^{2}-$ $c_{7} R$ becomes a constant. In any case, we have obtained a non-trivial polynomial 
relation among $P$ and $Q$ ! This argument works in the general situation and gives a proof of the above theorem. (Note that this argument does not work for the quasi-commuting pairs. See Section 7.)

Thus a pair of commuting operators produces a plane algebraic curve. Let us work out an explicit example due to Wallenberg. We choose

$$
\left\{\begin{array}{l}
P=\left(\frac{d}{d x}\right)^{2}-2 u(x) \\
Q=\left(\frac{d}{d x}\right)^{3}-3 u(x) \frac{d}{d x}-\frac{3}{2} u^{\prime}(x),
\end{array}\right.
$$

where $u^{\prime}(x)$ is the derivative of $u(x)$ with respect to $x$. It is an easy calculation to show that $P$ and $Q$ commute if and only if $u$ satisfies

$$
u^{\prime \prime \prime}=12 u u^{\prime} .
$$

We can integrate this equation twice to obtain

$$
\left(u^{\prime}\right)^{2}=4 u^{3}-g_{2} u-g_{3}
$$

where $g_{2}$ and $g_{3}$ are the constants of integration. Therefore, $P$ and $Q$ of $(2)$ commute if and only if their coefficients are given by the Weierstrass elliptic functions and their degenerations, since $g_{2}$ and $g_{3}$ in (3) are arbitrary. If $u$ satisfies (3), then $P$ and $Q$ satisfy a polynomial relation

$$
Q^{2}=P^{3}-\frac{g_{2}}{4} P-\frac{g_{3}}{4}
$$

which defines a plane cubic curve. It was already known to Wallenberg that every commuting pair of operators of order 2 and 3 can be reduced to this example by a simple transformation. In this sense this example is a universal one and the appearance of the elliptic function is essential.

When $P$ and $Q$ commute, the simultaneous eigenvalue problem

$$
\left\{\begin{array}{l}
P \psi=\lambda \psi \\
Q \psi=\mu \psi
\end{array}\right.
$$

makes sense. Since

$$
0=f(P, Q) \psi=f(\lambda, \mu) \psi
$$

the eigenvalues $\lambda$ and $\mu$ must satisfy the same polynomial relation. Therefore, the plane curve we obtained is a spectral curve. Moreover, a simple linear algebra argument shows that if $f(\lambda, \mu)=0$, then there exists a non-trivial solution of (4). If one uses the technique of asymptotic expansion of $\psi$, then it is not so hard to see that the dimension of the simultaneous eigenspace is equal to the greatest common divisor of the order of $P$ and $Q$. Thus, we can construct a vector bundle of rank $r=G . C . D .(\operatorname{ord} P, \operatorname{ord} Q)$ on the plane curve $\left\{(\lambda, \mu) \in \mathbb{C}^{2} \mid f(\lambda, \mu)=0\right\}$ 
whose fiber at $(\lambda, \mu)$ is the corresponding simultaneous eigenspace. There is a functorial way of extending this eigenspace bundle to the point at infinity of the spectral curve. Therefore, we obtain a compact Riemann surface by attaching a point at infinity to the plane curve and a globally defined vector bundle on it. In other words, we have constructed the data $(C, p, \mathcal{F})$ from the commutative algebra $B=\mathbb{C}[P, Q]$ generated by $P, Q$ and 1 , where $C$ is a compact Riemann surface which may have singularities, $p$ is the point at infinity and $\mathcal{F}$ is a vector bundle of rank $r=G . C . D .($ ord $P$, ord $Q)=\operatorname{rank} B$. In our example of (2), $C$ is an elliptic curve, $p$ is the point at infinity and $\mathcal{F}$ is a line bundle.

Let us denote by $\mathcal{M}_{1}$ the moduli space of all data $(C, p, \mathcal{L}, v)$ consisting of an algebraic curve of arbitrary genus $g$, a smooth point $p \in C$, a line bundle $\mathcal{L}$ on $C$ of degree $g-1$ which has no non-trivial global holomorphic sections, and a non-zero tangent vector $v \in T_{p} C$. (The tangent vector comes from the monicness assumption of the operator.) The second amazing theorem due to Burchnall and Chaundy states the following.

Second Theorem of Burchnall-Chaundy. Let $\mathcal{B}_{1}$ be the set of commutative algebras of ordinary differential operators of rank 1 satisfying (B-1), (B-2). Then there is a canonical bijection between $\mathcal{B}_{1}$ and $\mathcal{M}_{1}$;

$$
\beta_{1}: \mathcal{B}_{1} \stackrel{\sim}{\longrightarrow} \mathcal{M}_{1}
$$

These theorems were forgotten for more than half a century. Meantime, in the middle of 1970's, Krichever [K] re-discovered these theorems without knowing the earlier work of Burchnall and Chaundy. A complete proof of these theorems using the modern language of algebraic geometry was given by Mumford [Mum].

Here comes a natural question: which kind of geometric data correspond to $\mathcal{B}_{r}$ for $r \geq 2$ ?

An obvious candidate is the moduli space $\mathcal{M}_{r}$ of the data $(C, p, \mathcal{F}, v)$, where $\mathcal{F}$ is now a vector bundle of rank $r$ and degree $r(g-1)$ such that $H^{0}(C, \mathcal{F})=0$. But it does not work, because $\mathcal{B}_{r}$ is far larger than $\mathcal{M}_{r}$.

4. In order to prove the Main Theorem, we use the original technique of Burchnall and Chaundy as well as its modernized version due to Mumford. We also need another machinery, which is believed to be rather modern, but is actually very old: that is the theory of pseudo-differential operators.

Let us to go back to the history once again. I. Schur, inspired by the 1903 paper of Wallenberg, proved the following theorem in 1905. (In the same year he obtained also the famous Schur's lemma.)

Schur's Other Lemma. Let $B_{P}$ be the set of all linear ordinary differential operators which commute with a given operator $P$ of order $n>0$. Then $B_{P}$ is a commutative algebra. 
The theorem is rather surprising, because it does not hold for the case of matrices and partial differential operators. Schur's proof is the following. As before, we can assume that $P$ is monic. The first thing he did is to define the $n$-th root of $P$. He showed that there is a unique monic pseudo-differential operator

$$
L=\frac{d}{d x}+a_{0}+a_{1}\left(\frac{d}{d x}\right)^{-1}+a_{2}\left(\frac{d}{d x}\right)^{-2}+\cdots
$$

of order one such that $L^{n}=P$. (He credited S. Pincherle $[\mathrm{P}]$, which appeared in 1897, for the technique of pseudo-differential calculus. The idea of fractional powers of operators is due to Schur himself.) Let $Q \in B_{P}$ be an arbitrary operator of order, say $m$. Since $Q$ commutes with $P$, its leading coefficient is a constant. Therefore, there is a constant $c_{0} \in \mathbb{C}$ such that $Q-c_{0} L^{m}$ has order $m-1$ or less. Since $L$ commutes with $P, Q-c_{0} L^{m}$ also commutes with $P$. Therefore, its leading coefficient is again a constant, and hence there is $c_{1} \in \mathbb{C}$ so that $Q-c_{0} L^{m}-c_{1} L^{m-1}$ is of order $m-2$ or lower. By continuing this process infinitely many times, he obtained

$$
Q=\sum_{\ell=0}^{\infty} c_{\ell} L^{m-\ell}
$$

Therefore,

$$
B_{P} \subset \mathbb{C}\left(\left(L^{-1}\right)\right),
$$

where $\mathbb{C}((z))$ denotes the set of all formal Laurent series in $z$ with finite poles at $z=0$. But since $\mathbb{C}\left(\left(L^{-1}\right)\right)$ is commutative, so is $B_{P}$ !

Let us consider the abstract version of Schur's argument. Since $Q$ is a differential operator, $m$ of (6) is always positive. Thus we have a subalgebra

$$
A \subset \mathbb{C}((z))
$$

satisfying the condition

$$
A \cap \mathbb{C}[[z]]=\mathbb{C} .
$$

A commutative algebra $B$ satisfying (B-1) and (B-2) produces a pair $(A, L)$, where $L$ is a monic pseudo-differential operator of order 1 obtained by taking the $n$-th root of $P$, and $A$ is a subalgebra of $\mathbb{C}((z))$ with $(9)$ obtained by replacing $L^{-1}$ in (6) by $z$.

Now the question: how can we go back from an abstract algebra $A$ satisfying (8) and (9) to the algebra $B$ of ordinary differential operators?

An easy answer is this: simply replace $z$ by $L^{-1}$. But then how do we know that the resulting algebra consists of only differential operators? In other words, how can we find an operator $L$ so that the algebra

$$
B=\left\{a\left(L^{-1}\right) \mid a(z) \in A\right\}
$$


obtained by replacing $z$ by $L^{-1}$ in $A$ contains only differential operators? In order to answer this question, we need another idea.

5. In their fundamental work [GD] on completely integral systems appeared in 1975 and 76, Gel'fand and Dikii introduced negative and fractional powers of operators in this field. But actually the same technique had been known to Pincherle and Schur for more than 70 years before. Thus many of these modern theories on this subject have deep historical background, even though it was not recognized until recently. Then M. Sato [Sa] discovered a remarkable correspondence between an infinite dimensional Grassmannian and the pseudo-differential operators of Schur and Gel'fand-Dikii.

We have already encountered the set $\mathbb{C}((z))$ of all formal Laurent series. Let $V=\mathbb{C}((z))$ and $V^{(\nu)}=\mathbb{C}[[z]] \cdot z^{-\nu}$. Then the filtration

$$
\cdots \supset V^{(\nu+1)} \supset V^{(\nu)} \supset V^{(\nu-1)} \supset \cdots
$$

defines a topology in $V$. We define the Grassmannian $G(\mu, \nu)$ of index $\mu$ and level $\nu$ as the set of all closed vector subspaces $W$ of $V$ such that both $W \cap V^{(\nu)}$ and $V /\left(W+V^{(\nu)}\right)$ have finite dimensions and

$$
\operatorname{dim}\left(W \cap V^{(\nu)}\right)-\operatorname{dim}\left(V /\left(W+V^{(\nu)}\right)\right)=\mu
$$

This is indeed the Grassmannian of Fredholm operators of index $\mu$. The big-cell of the index 0 Grassmannian is defined by

$$
G^{+}(0, \nu)=\left\{W \in G(0, \nu) \mid W \cap V^{(\nu)}=V /\left(W+V^{(\nu)}\right)=0\right\}
$$

In 1981, Sato found a bijective correspondence between the Grassmannian $G(0,0)$ and the set of monic pseudo-differential operators

$$
S=1+s_{1}(x)\left(\frac{d}{d x}\right)^{-1}+s_{2}(x)\left(\frac{d}{d x}\right)^{-2}+\cdots
$$

of order zero which have certain type of singularities in their coefficients $s_{j}(x)$. In particular, if $s_{j}(x)$ 's are all regular at $x=0$, i.e. $s_{j}(x) \in \mathbb{C}[[x]]$ for all $j \geq 1$, then $S$ of (10) corresponds bijectively to a point of the big-cell $G^{+}(0,0)$. Unfortunately this correspondence is not canonical, because it depends on the choice of a basis for $V$. The corresondence becomes completely canonical if one uses $G^{+}(0,-1)$ instead of $G^{+}(0,0)$. Thus one obtains the following.

Theorem 1. Let $\Gamma_{m}$ be the set of all monic zeroth order pseudo-differential operators of the form (10) such that all the coefficients are in $\mathbb{C}[[x]]$. Then there is a canonical bijection

$$
\Gamma_{m} \stackrel{\sim}{\longrightarrow} G^{+}(0,-1)
$$


We can choose an invertible function $f \in \mathbb{C}[[x]]$ so that Schur's operator $L$ of (5) becomes

$$
f \cdot L \cdot f^{-1}=\frac{d}{d x}+0+u_{1}(x)\left(\frac{d}{d x}\right)^{-1}+u_{2}(x)\left(\frac{d}{d x}\right)^{-2}+\cdots
$$

Then there is an operator $S \in \Gamma_{m}$ such that $S \cdot \frac{d}{d x} \cdot S^{-1}$ is equal to the above expression. Therefore, if we start with $f \cdot B \cdot f^{-1}$ instead of $B$ (and which we identify anyway by (1)), then we have an operator $P \in B$ such that $S^{-1} \cdot P \cdot S=\left(\frac{d}{d x}\right)^{n}$. It is easy to see that every operator commuting with $\left(\frac{d}{d x}\right)^{n}$ for some $n \geq 1$ has only constant coefficients. Therefore, we have

$$
A=S^{-1} B S \subset \mathbb{C}\left(\left(\partial^{-1}\right)\right)=\mathbb{C}((z))
$$

where $\partial=\frac{d}{d x}$ and we identify $z=\partial^{-1}$. Note that "replacing $z^{-1}$ by $L$ " simply means that changing $\partial$ by $S \partial S^{-1}=L$. Thus the operator $S \in \Gamma_{m}$ connects the algebra $B$ and the abstract algebra $A \subset \mathbb{C}((z))$. So we can replace the pair $(A, L)$ by another pair $(A, W)$, where $W \in G^{+}(0,-1)$ is the point of the Grassmannian corresponding to $S$. We call $(A, W)$ a Schur pair.

6. A connection between the Grassmannian and the geometric data consisting of pointed algebraic curves and vector bundles on them was discovered by G. Segal and G. Wilson [SW] in 1983. They dealt with the Grassmannian from the point of view of loop groups. Hence they used the Hilbert space $H^{(r)}$ of $\mathbb{C}^{r}$-valued square integrable functions on a circle in order to obtain an extended moduli space of curves and vector bundles of rank $r$. Then they defined an isomorphism $H^{(r)} \stackrel{\sim}{\longrightarrow} H^{(1)}$ and brought the higher rank situation to the single Grassmannian, which is the analytic version of Sato's $G(0,0)$. However, the isomorphism between $H^{(r)}$ and $H^{(1)}$ is not canonical.

In order to find a canonical bijection between the data of the Grassmannians and the geometric data of pointed curves and vector bundles, we use Schur pairs. We call $(A, W)$ a Schur pair of rank $r$, index $\mu$ and level $\nu$ if

1. $W \in G(\mu, \nu)$ and

2. $\mathbb{C} \subset A \subset V$ is a subalgebra such that $A W \subset W$ by multiplication of $V=\mathbb{C}((z))$ and

$$
r=\operatorname{rank} A=G . C . D .\{\text { pole order of } a(z) \text { at } z=0 \mid a(z) \in A\} .
$$

Let $\mathcal{S}_{r}(\mu, \nu)$ denote the set of all Schur pairs of rank $r$, index $\mu$ and level $\nu$.

The geometric counterpart of the set of Schur pairs is the moduli space $\mathcal{M}_{r}(\mu, \nu)$ of quintets $(C, p, \mathcal{F}, \pi, \phi)$, where $C, p$ and $\pi$ are as in Section $2, \mathcal{F}$ is now an arbitrary torsion free sheaf of rank $r \mathcal{O}_{C}$-modules such that

$$
\operatorname{dim} H^{0}(C, \mathcal{F})-\operatorname{dim} H^{1}(C, \mathcal{F})=\mu
$$


and

$$
\phi:\left.\mathcal{F}\right|_{U_{p}} \stackrel{\sim}{\longrightarrow} \pi_{*} \mathcal{O}_{U_{0}}(\nu)
$$

is an $\mathcal{O}_{U_{p}}$-module isomorphism. Note that in the case of rank one, $\pi$ gives a local coordinate on $U_{p}$ and hence our quintet becomes the quintuple of Segal-Wilson.

We have the following theorem.

Theorem 2. There is a canonical bijection

$$
\chi_{r, \mu, \nu}: \mathcal{M}_{r}(\mu, \nu) \stackrel{\sim}{\longrightarrow} \mathcal{S}_{r}(\mu, \nu)
$$

for every $r>0, \mu \in \mathbb{Z}$ and $\nu \in \mathbb{Z}$. Moreover, there are the following canonical isomorphism under this correspondence:

$$
\begin{aligned}
& H^{0}(C, \mathcal{F}) \simeq W \cap V^{(\nu)} \quad \text { and } \\
& H^{1}(C, \mathcal{F}) \simeq V /\left(W+V^{(\nu)}\right) .
\end{aligned}
$$

Roughly speaking, $\chi_{r, \mu, \nu}(C, p, \mathcal{F}, \pi, \phi)=(A, W)$ is defined by

$$
\begin{aligned}
A & =\text { holomorphic functions on } C \backslash\{p\}, \text { and } \\
W & =\text { holomorphic sections of }\left.\mathcal{F}\right|_{C \backslash\{p\}} .
\end{aligned}
$$

In [M3], this bijection is obtained as a fully faithful functor between anti-equivalent categories.

As the final step to prove the main theorem, we need the following

Theorem 3. Let $(A, W) \in \mathcal{S}_{r}(0,-1)$ be a Schur pair of rank $r$, index 0 and level -1 such that $W$ belongs to the big-cell $G^{+}(0,-1)$ of the Grassmannian, and let $S \in$ $\Gamma_{m}$ be the pseudo-differential operator corresponding to the point $W$ by the Sato correspondence (Theorem 1). Then $B=S \cdot A \cdot S^{-1}$ is a rank $r$ commutative algebra of ordinary differential operators, where we regard $A$ as a subring of $\mathbb{C}\left(\left(\partial^{-1}\right)\right)$ by the identification $z=\partial^{-1}$.

Let $D$ denote the set of all linear ordinary differential operators with coefficients in $\mathbb{C}[[x]]$. Then for a given algebra $A$ with (8) and (9), the condition $S \cdot A \cdot S^{-1} \subset D$ gives a system of nonlinear ordinary differential equations among the coefficients of $S$ which was studied in [M2, Section 1]. Theorem 3 tells us that this system has a regular solution if and only if there is a point $W \in G^{+}(0,-1)$ such that $A W \subset W$.

Schur showed that every $B \in \mathcal{B}_{r}$ gives a pair $(A, L)$ and hence, by Theorem 1 , the Schur pair $(A, W) \in \mathcal{S}_{r}(0,-1)$. Now Theorem 3 tells us how to go back from $(A, W)$ to $B$. On the other hand, we have a bijection $\chi_{r, \mu, \nu}$ of (11) by Theorem 2. Combining these two constructions, we obtain the main theorem.

One of the most important motivations of studying commuting ordinary differential operator is their relation with completely integrable systems, and this point of 
view of the problem is entirely missing in the classical works I mentioned above. For the recent deep works on commuting operators and completely integrable systems, I refer to two books [N] and [NMPZ].

A detailed discussion of the topics of this paper is given in [M3].

7. As we saw in Section 3, one of the key steps of our classification theorem is the Burchnall-Chaundy theory: $[P, Q]=0$ implies a polynomial relation. Being motivated by the recent theory of two dimensional quantum gravity, a natural question arises here: what happens is we have quasi-commutativity $[P, Q] \equiv 1$ ?

Certainly, the argument of Section 3 does not apply.

Theorem 4. Let $P$ and $Q$ be linear ordinary differential operators satisfying the quasi-commutativity condition

$$
[P, Q]=c=\text { constant }
$$

If $c \neq 0$, then the operators cannot satisfy any non-trivial relation of the form

$$
\sum_{i, j}^{\text {finite }} c_{i j} P^{i} Q^{j}=0
$$

with constant coefficients.

Proof. Let

$$
f(X, Y)=\sum_{i, j}^{\text {finite }} c_{i j} X^{i} Y^{j}
$$

be a polynomial such that $f(P, Q) \equiv 0$ in this order. If this equation is non-trivial with respect to $Q$, then we can choose such a polynomial with the lowest degree in $Q$. Note that $[P, f(P, Q)]=0$, i.e.

$$
\sum_{i, j}^{\text {finite }} c_{i j} c j P^{i} Q^{j-1}=0 .
$$

But this equation has a lower degree in $Q$, hence $f(X, Y)$ does not depend on $Y$.

This is the point where the quantum and the non-commutative geometry start, but we have to stop this article here.

\section{References}

[BC] J.L. Burchnall and T.W. Chaundy: Commutative ordinary differential operators, Proc. London Math. Soc. Ser. 2, 21 (1923) 420-440; Proc. Royal Soc. London Ser. A, 118 (1928) 557-583. 
[F] G. Floquet: Sur la théorie des équations différentielles linéaires, Ann. Sci. de l'École Norm. Supér., 8 (1879) Suppl., 3-132.

[GD] I.M. Gel'fand and L.A. Dikii: Asymptotic behavior of the resolvent of Sturm-Liouville equations and the algebra of the Korteweg-de Vries equations, Russ. Math. Surveys, 30 (1975) 77-113; Fractional powers of operators and Hamiltonian systems, Func. Anal. Appl. 10 (1976) 259-273.

[K] I.M. Krichever: Methods of algebraic geometry in the theory of nonlinear equations, Russ. Math. Surveys 32 (1977) 185-214.

$[\mathrm{Ku}] \quad$ M. Kuga: Galois' Dream, (translated by S. Addington from the Japanese text), Birkhäuser, to appear.

[M1] M. Mulase: Algebraic geometry of soliton equations, Proc. Japan Acad. Ser. A, 59 (1983) 285-288.

[M2] _ Cohomological structure in soliton equations and jacobian varieties, J. Diff. Geom. 19 (1984) 403-430.

[M3] _ Category of vector bundles on algebraic curves and infinite dimensional Grassmannians, Intern. Journ. Math. 1 (1990) to appear.

[Mum] D. Mumford: An algebro-geometric constructions of commuting operators and of solutions to the Toda lattice equations, Korteweg-de Vries equations and related non-linear equations, In Proc. Internat. Symp. on Alg. Geom., Kyoto 1977, Kinokuniya Publ. (1978) 115-153.

[N] S.P. Novikov, ed.: Integrable systems, London Math. Soc. Lec. Notes Ser. 60, Cambridge Univ. Press (1981).

[NMPZ] S.P. Novikov, S.V. Manakov, L.P. Pitaevskii and V.E. Zakharov: Theory of solitons - The inverse scattering method, Consultants Bureau, New York (1984).

[P] S. Pincherle: Mémoire sur le calcul fonctionnel distributif, Math. Ann. 49 (1897) 325-382.

$[\mathrm{PW}] \quad$ E. Previato and G. Wilson: Vector bundles over curves and solutions of the KP equations, Proc. Symp. Pure Math. 49 (1989) 553-569.

[Sa] M. Sato: Soliton equations as dynamical systems on an infinite dimensional Grassmannian manifold, Kokyuroku, Res. Inst. Math. Sci., Kyoto Univ. 439 (1981) 30-46.

[SN] M. Sato and M. Noumi: Soliton equations and universal Grassmann manifold (in Japanese), Sophia Univ. Lec. Notes Ser. in Math. 18 (1984).

[Sc] I. Schur: Über vertauschbare lineare Differentialausdrücke, Sitzungsber. der Berliner Math. Gesel. 4 (1905) 2-8. 
[SW] G.B. Segal and G. Wilson: Loop groups and equations of $K d V$ type, Publ. Math. I.H.E.S. 61 (1985) 5-65.

[V] J.-L. Verdier: Equations differentielles algébriques, Séminaire de lÉcole Normale Supérieure 1979-82, Birkhäuser (1983) 215-236.

[W] G. Wallenberg: Über die Vertauschbarkeit homogener linearer Differentialausdrücke, Archiv der Math. u. Phys., Drittle Reihe 4 (1903) 252-268. 